\title{
Network calculations for cosmochronometric studies
}

\author{
I. Petermann \\ University of Malaya, 50603 Kuala Lumpur, Malaysia \\ Email: $\underline{\text { ilka@um.edu.my }}$
}

(Received Feb 2011; Published March 2011)

\begin{abstract}
The determinations of the age of the oldest objects in the universe, giving a lower limit of the age of the universe itself, are of great interest in astrophysics and cosmology. With the detection of the long-lived isotopes of thorium and uranium in a single star, their application as cosmochronometers has become possible. With network calculations, the production of Th and U can be studied for different input of the underlying mass model and the astrophysical environment. A comparison with the abundance pattern of very metal-poor stars can serve as a reference to check the validity of the assumptions and to better understand their influences on the final outcomes of the calculations. Once the initial amount of thorium and uranium are known, age estimations of stellar objects can be accomplished. Network calculations were performed that allow flexibly changing and studying the astrophysical environment in a parametric way, as well as the underlying nuclear physics input in terms of the final abundance pattern. The network used here includes more than 7000 nuclei, reaching far outside in the neutron rich area. The most relevant fission processes were implicitly taken into account. For all three massmodels a good agreement between the final abundance pattern and the abundance distribution for six metal poor stars is demonstrated. Network calculations are thus an appropriate tool to study and analyze the production of the cosmochronometers Th and U, which can be used for age-determinations for stars. A more detailed study of their production paths is necessary to reduce uncertainties with respect to the underlying massmodel and astrophysical environment.
\end{abstract}

Key words: cosmochronometry, metal-poor stars, r-process

https://doi.org/10.14331/ijfps.2011.330004

\section{INTRODUCTION}

Determining the age of the oldest stars in the universe gives the opportunity to set a lower limit of the age of the universe. If the long-lived isotopes of thorium and uranium (with lifetimes of $\tau\left({ }^{232} \mathrm{Th}\right)=2.03 \cdot 10^{10}$ years and $\left.\tau^{(238} \mathrm{U}\right)=6.45 \cdot 10^{9}$ years $)$ can be detected in one stellar object, they can be used for cosmochronometric studies. Using these two neighbouring nuclei, a reduction of uncertainties concerning their nuclear properties and production paths was commonly assumed, albeit using stable isotopes co-produced in the $r$-process, such as $\mathrm{Eu}$, is also widely applied. The age of the star can be determined when assuming a purely exponential decay (Cayrel et al., 2001) of the radioactive isotopes as $(\mathrm{U} / \mathrm{Th})_{\mathrm{obs}}=(\mathrm{U} / \mathrm{Th})_{\mathrm{ini}} \cdot \exp \left[-\mathrm{t}^{*} \cdot \tau\left(1 /\left({ }^{238} \mathrm{U}\right)\right.\right.$ $\left.\left.-1 / \tau\left({ }^{232} \mathrm{Th}\right)\right)\right]$. The crucial point in this analysis is the availability of initial abundances of the cosmochronometers, which is accomplished by theoretical calculations. It is known that heavy neutronrich nuclei, such as $\mathrm{Th}, \mathrm{U}$ and their progenitors, are produced in the $r$-process. The $r$-process is a series of $r$ apid neutron capture reactions and successive $\beta$-decays in explosive scenarios with extremely high neutron densities. Although its astrophysical site has not been unambiguously identified, supernovae of type II are commonly accepted as a very promising site.

\section{METHOD}

As the environmental conditions do not allow a description via a nuclear statistical equilibrium, a system of network equations has to be solved to describe the evolution of the abundances for each isotope with time. Separating the reactions in terms of the number of reactants, the nuclear network is given by the set of differential equations,

$$
\dot{Y}_{i}=\sum_{j} N_{j}^{i} Y_{i}+\sum_{j, k} N_{j, k}^{i} \rho N_{A}\langle j, k\rangle Y_{j} Y_{k}+\sum_{j, k, l} N_{j, k, l}^{i} \rho^{2} N_{A}^{2}\langle j, k, l\rangle Y_{j} Y_{k} Y_{l}
$$

describing the evolution of the abundance $Y_{i}$ of species $i$. The density is given by $\rho, \mathrm{N}_{\mathrm{A}}$ is the Avogadro number. Writing this equation for those processes that are known to dominate the $r$ - 
process, one arrives at the network equation with the abundances $\mathrm{Y}$ for nuclei with proton (mass) number $\mathrm{Z}(\mathrm{A})$, cross section $\sigma$ and decay constant $\lambda$ as

$$
\begin{aligned}
& \frac{d Y_{(A, Z)}}{d t}=-\lambda_{m}(A, Z) Y_{(A, Z)}-\rho(t) N_{A} Y_{N} Y_{(A, Z)}\left\langle\sigma_{n \gamma}(A, Z) v\right\rangle \\
& +\rho(t) N_{A} Y_{n} Y_{(A-1, Z)}\left\langle\sigma_{n \gamma}(A-1, Z) v\right\rangle \\
& +\lambda_{m}(A+1, Z) Y_{(A+1, Z)} \\
& -\lambda_{\alpha}(A, Z) Y_{(A, Z)}+\lambda_{\alpha}(A+4, Z+2) Y_{(A+4, Z+2)} \\
& +\lambda_{\beta}(A, Z-1) Y_{(A, Z-1)}-\lambda_{\beta}(A, Z) Y_{(A, Z)} \\
& -\rho(t) N_{A} Y_{n} Y_{(A, Z)}\left\langle\sigma_{n f}(A, Z) v\right\rangle-\lambda_{\beta f}(A, Z) Y_{(A, Z)}-\lambda_{s f}(A, Z) Y_{A, Z}
\end{aligned}
$$

The last three terms denote neutron-induced, $\beta$-delayed and spontaneous fission that are known to be very important processes for heavy (neutron-rich) nuclei reached by the $r$ process.

The largest fractions of nuclei relevant for the $r$-process are located far on the neutron-rich region. They are not yet accessible by experiment; hence theoretical descriptions have to be applied to determine the relevant quantities. The masses, as the basic ingredient, were taken in this work from the Extended Thomas-Fermi plus Strutinsky-Integral (ETFSI, (Pearson et al., 2006)), Finite-Range Droplet model (FRDM, (Möller et al., 1995)), and Hartree-Fock-Bogolyubov approach (HFB, (Goriely et al., 2001)). The masses are then used to determine neutroncapture rates, $\alpha-/$ and $\beta$-decays as well as neutron-induced, $\beta$ delayed and spontaneous fission rates. For the fission rates (Panov et al., 2005), the corresponding yields together with the number of evaporated neutrons were given by (Kelic et al., 2009). With these calculations, a pure $r$-process abundance pattern is created. To compare with observed data it would be desirable to detect those stars in nature, which were built from material which was enriched only by a few, or even only one, supernova event. These stars have to be naturally very old. A common way to express the age of the star is by the stellar metallicity, using the abundance of the end-product of stellar burning of massive stars, iron, compared to hydrogen, $[\mathrm{Fe} / \mathrm{H}]$ using the common definition $[\mathrm{A} / \mathrm{B}]=\log _{10}\left(\mathrm{~N}_{\mathrm{A}} / \mathrm{N}_{\mathrm{B}}\right)_{\mathrm{star}}-$ $\log _{10}\left(\mathrm{~N}_{\mathrm{A}} / \mathrm{N}_{\mathrm{B}}\right)_{\text {solar }}$.

In the last years, a growing amount of metal-poor stars has been observed. However, the total amount of detected stars with very low metallicity is still very small. When comparing the (scaled) solar-system distribution to the abundance pattern of metal-poor stars (Sneden et al., 2000), a considerable agreement for elements with proton numbers $56<\mathrm{Z}<70$ is found. Differences are mostly seen in the range below $Z=56$, suggesting further investigations concerning the production paths of nuclei in these lighter and heavier mass regions. Despite this agreement, that seems to indicate a unique site of the $r$-process, the material the solar-system is composed of, has experienced enrichment by heavy elements of many supernova events.

Therefore, comparisons of the outcomes of network-calculations to the solar-system abundance pattern are convenient; however distributions of metal-poor stars would be preferred. Today, only three stars are known to have $[\mathrm{Fe} / \mathrm{H}]<-4.0$. The first star found in 1984 by Bessell and Norris (Bessell and Norris, 1984), CD$38^{\circ} 245$, was described to have $[\mathrm{Fe} / \mathrm{H}]=-4.0$. More than twenty years later, in 2007, the discovery of a second object with $[\mathrm{Fe} / \mathrm{H}]<4.8$, HE 0557-4840, was reported (Norris et al. 2007). Well below that value was the star HE0107-5240 described in 2002 (Christlieb et al. 2002) with a value of $[\mathrm{Fe} / \mathrm{H}]$ as low as 5.3. Very recently an even lower metallicity, $[\mathrm{Fe} / \mathrm{H}]=-5.4$ was reported in 2005 (Frebel et al., 2005) for HE1327-2326.

For cosmochronometric studies, the stars have to additionally show an increased amount of $r$-process elements. In most cases the ratio of $\mathrm{Th}$ to $\mathrm{Eu}$ is applied, as both elements are solely produced in the $r$-process and were relatively easy to detect in various metal-poor stars. However, they differ considerably in their proton number and using abundance ratios of isotopes lying in closer vicinity to each other has the advantage that one can assume that they have rather similar nuclear properties.

Therefore their production mechanisms should resemble each other to a good extent, thus reducing uncertainties due to the nuclear physics input. Therefore, using the ratio of Th to $\mathrm{U}$ would be an appropriate choice. However, U has up to now only be detected in three metal-poor objects: With the spectroscopic analysis of the giant star CS 31082-001 in 2001 an uranium abundance was given for the first time, followed by second object, CS29497-004, (Christlieb et al., 2004). In 2007, a third star where uranium could be determined is the object HE15230901, discovered by (Frebel et al., 2007). Showing a metallicity of only $[\mathrm{Fe} / \mathrm{H}]=-2.95$ it is in addition highly enhanced in $r$ process elements. The inclusion of $U$ gives rise to the pairs of chronometers $\mathrm{Th} / \mathrm{U}$, as well as $\mathrm{U} / \mathrm{Os}$, resulting in an average age of 13.2 Gyr for this star.

\section{RESULTS AND DISCUSSION}

With network calculations it is possible to calculate the initial abundances of the cosmochronometers (and their progenitors). In addition, instead of assuming their purely exponential decay with time, their abundances can be followed over the complete evolution till the latest times. In Fig. 1 a comparison between network calculations applying the massmodels FRDM, ETFSI and HFB and six metal-poor, namely HD110184 (Honda et al., 2004), HD6268 (Cowan et al., 2005), CS22892-052 (Sneden et al., 2003) and HD122563, HD115444, HD186478 (all three (Simmerer et al., 2004)), is given. Abundances (here after $1 \mathrm{Gyr}$ ) were normalized to $\mathrm{Eu}$, as it is assumed to be produced by roughly $95 \%$ during the $r$-process (Arlandini et al., 1999) .The overall agreement between the calculated abundance pattern and the different stellar objects shows a good agreement. For Z=77 the value given by FRDM, having the largest abundance in the given region when compared to ETFSI and HFB, lies much closer to the value that are given in the stars HD115444 and HD6268, which need further investigation in terms of the production path. With FRDM the amount of Th and $U$ is overestimated for all given cases, while with ETFSI the values 
as given for HD6268 and CS22892-052 are reproduced with good agreement.

For the other three cases shown in Fig. 1 the situation is less clear, although the results obtained with HFB, as lying between the result of ETFSI and FRDM, are lying closest to the given data. To understand this behaviour, more detailed studies investigating the different productions paths of the cosmochronometers will be done. However, it was shown that the overall structure for a broad range of nuclei is clearly reproduced. Future attempts will be made towards a more detailed understanding of the influence of the underlying massmodel as well as the astrophysical environment. In a more detailed analysis a purely exponential decay is questioned and it is investigated if a broader range of nuclei might contribute to the built-up of thorium and uranium (PhD Petermann, 2010), affecting the age determinations of stars.

\section{REFERENCES}

C. Arlandini, F. Käppeler, K. Wisshak, R. Gallino, M. Lugaro, M. Busso, and O. Straniero, 1999. Neutron Capture in Low-Mass Asymptotic Giant Branch Stars: Cross Sections and Abundance Signatures. Astrophysical Journal, 525:886-900.

M. S. Bessell and J. Norris, 1984. The ultra-metal-deficient (Population III?) red giant CD 38.245 deg. Astrophysical Journal, 285:622-636.

R. Cayrel, V. Hill, T. C. Beers, B. Barbuy, M. Spite, F. Spite, B. Plez, J. Andersen, P. Bonifacio, P. François, P. Molaro, B. Nordström, and F. Primas, 2001. Measurement of stellar age from uranium decay. Nature, 409:691-692.

N. Christlieb, M. S. Bessell, T. C. Beers, B. Gustafsson, A. Korn, P. S. Barklem, T. Karlsson, M. Mizuno-Wiedner, and S. Rossi, 2002. A stellar relic from the early Milky Way. Nature, 419:904906.

N. Christlieb, B. Gustafsson, A. J. Korn, P. S. Barklem, T. C. Beers, M. S. Bessell, T. Karlsson, and M. Mizuno-Wiedner, 2004. HE 0107-5240, a Chemically Ancient Star. I. A Detailed Abundance Analysis. Astrophysical Journal, 603:708-728.

J. J. Cowan, C. Sneden, T. C. Beers, J. E. Lawler, J. Simmerer, J. W. Truran, F. Primas, J. Collier, and S. Burles, 2005. Hubble Space Telescope Observations of Heavy Elements in Metal-Poor Galactic Halo Stars. Astrophysical Journal, 627:238-250.

A. Frebel, W. Aoki, N. Christlieb, H. Ando, M. Asplund, P. S. Barklem, T. C. Beers, K. Eriksson, C. Fechner, M. Y. Fujimoto, S. Honda, T. Kajino, T. Minezaki, K. Nomoto, J. E. Norris, S. G. Ryan, M. Takada-Hidai, S. Tsangarides, and Y. Yoshii, 2005. The new record holder for the most ironpoor star: HE 1327 2326, a dwarf or subgiant with $[\mathrm{Fe} / \mathrm{H}]=-5.4$. In V. Hill, P. François, \& F. Primas, editor, From Lithium to Uranium: Elemental Tracers of Early Cosmic Evolution, volume 228 of IAU Symposium, pages 207-212.
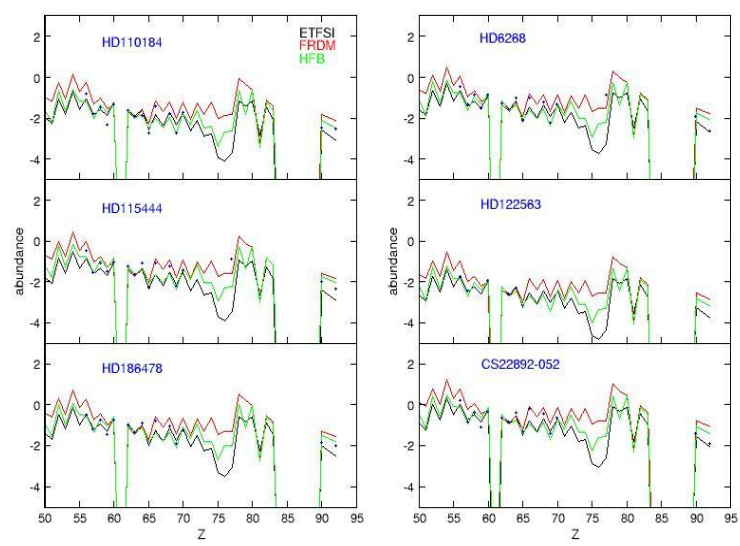

Figure 1: Calculations for ETFSI, FRDM and HFB for an entropy $\mathrm{s}=250$ and electron-fraction $\mathrm{Y}_{\mathrm{e}}=0.40$ compared to metalpoor stars; abundances are normalized to $\mathrm{Eu}$

A. Frebel, N. Christlieb, J. E. Norris, C. Thom, T. C. Beers, and J. Rhee, 2007. Discovery of HE 1523-0901, a Strongly rProcess-enhanced Metal-poor Star with Detected Uranium. Astrophysical Journal, 660:L117-L120.

S. Goriely, F. Tondeur, and J. M. Pearson, 2001. A Hartree-Fock Nuclear Mass Table. Atomic Data and Nuclear Data Tables, 77:311-381.

S. Honda, W. Aoki, T. Kajino, H. Ando, T. C. Beers, H. Izumiura, K. Sadakane, and M. Takada-Hidai, 2004. Spectroscopic Studies of Extremely Metal-Poor Stars with the Subaru High Dispersion Spectrograph. II. The r-Process Elements, Including Thorium. Astrophysical Journal, 607:474498.

A. Keliç, M. Valentina Ricciardi, and K.-H. Schmidt. ABLA07 - towards a complete description of the decay channels of a nuclear system from spontaneous fission to multifragmentation, 2009. ArXiv e-prints, 2009arXiv0906.4193K.

P. Möller, J. R. Nix, W. D. Myers, and W. J. Swiatecki. Nuclear Ground-State Masses and Deformations, 1995. Atomic Data and Nuclear Data Tables, 59:185-+.

J. E. Norris, N. Christlieb, A. J. Korn, K. Eriksson, M. S. Bessell, T. C. Beers, L. Wisotzki, and D. Reimers. HE 0557-4840: UltraMetal-Poor and Carbon-Rich, 2007. Astrophysical Journal, 670:774-788.

I. V. Panov, E. Kolbe, B. Pfeiffer, T. Rauscher, K.-L. Kratz, and F.-K.Thielemann. Calculations of fission rates for $\mathrm{r}$-process nucleosynthesis, 2005. Nuclear Physics A, 747:633-654.

J. M. Pearson and S. Goriely. Nuclear mass formulas for astrophysics, 2006. Nuclear Physics A, 777:623-644.

I.Petermann (2010) Influence of Fission Processes on r-Process Network Calculations. PhD Dissertation, TU Darmstadt, Germany.

J. Simmerer, C. Sneden, J. J. Cowan, J. Collier, V. M. Woolf, and J. E. Lawler. The Rise of the s-Process in the Galaxy, 2004. Astrophysical Journal, 617:1091-1114. 
C. Sneden, J. J. Cowan, I. I. Ivans, G. M. Fuller, S. Burles, T. C. Beers, and J. E. Lawler. Evidence of Multiple R-Process Sites in the Early Galaxy: New Observations of CS 22892-052, 2000. Astrophysical Journal, 533:L139-L142.
C. Sneden, J. J. Cowan, J. E. Lawler, I. I. Ivans, S. Burles, T. C. Beers, F. Primas, V. Hill, J. W. Truran, G. M. Fuller, B. Pfeiffer, and K.-L. Kratz. The Extremely Metal-poor, Neutron Capturerich Star CS 22892-052:A Comprehensive Abundance Analysis, 2003. Astrophysical Journal,591:936-953. 